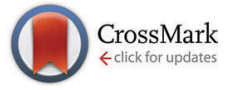

Cite this: Phys. Chem. Chem. Phys., 2016, 18, 4221

DOI: 10.1039/c6cp90016j

www.rsc.org/pccp

\title{
Correction: Singlet lifetime measurements in an all-proton chemically equivalent spin system by hyperpolarization and weak spin lock transfers
}

\author{
Y. Zhang, K. Basu, J. W. Canary and A. Jerschow* \\ Correction for 'Singlet lifetime measurements in an all-proton chemically equivalent spin system by \\ hyperpolarization and weak spin lock transfers' by Y. Zhang et al., Phys. Chem. Chem. Phys., 2015, 17, \\ 24370-24375.
}

\begin{abstract}
On page 24371, left column, the calculated coupling constants have been reported lower by a factor of two by mistake. The sentence should read: This calculation gave the couplings between the vinylene protons as $16.6 \mathrm{~Hz}$ and the out of pair couplings as $-0.26 \mathrm{~Hz}$ and $-0.10 \mathrm{~Hz}$.
\end{abstract}

This does not affect the rest of the manuscript, because only the experimentally determined values were used later.

The Royal Society of Chemistry apologises for these errors and any consequent inconvenience to authors and readers. 